analysierende Menge des vorher ins Chlorid übergeführten Metalls unter Amylalkohol cingedampft und die Losung durch einen. Goochtiegel abgesaugt. Der Rückstand war total in Wasser löslich; er wurde in einer Platinschale zur Wägung gebracht. $0,12 \mathrm{~g}$ Metall ergaben einen Rückstand von $0,0005 \mathrm{~g}$ Natriumchlorid $=0,17 \% N a$. Dieser Natriumgehalt entstammt dem Ausgangsmaterial.

Da über den Schmelzpunkt des Lithiums neben einer Angabe von Bunsen ${ }^{1}$ ) nur noch eine neuere von $\mathrm{Kahlbaum}^{2}$ ) vorliegt, haben wir diesen erneut bestimmt. Während Kahlbaum das Lithium in einem Paraffinbad zum Schmelzen erhitzte und die Temperatur des letzteren beobachtete, also eine Ueberhitzung des Bades kaum vermeiden konnte, haben wir das Thermometer direkt in das Lithium eingetaucht. Das Instrument steckte in einer dünnen Stahlhülse, während sich das Lithium - es waren gegen I $5 \mathrm{~g}-$ in einem eisernen Tiegel befand, der zur Vermeidung von Oxydation des

1) Lieb. Ann. d. Chemie 94, IO7 (I855); siehe auch Borchers, Elektrometallurgie, S. 20 .

2) Zeitschr. f. atorg. Chemie 23, 220 (IģOo).
Metalles in ein mit der Quecksilberluftpumpe evalkuiertes Glasrohr eingeschmolzen war. Das Ganze befand sich in einem grossen Oelbad mit Rührer.

Die Temperaturen des Schmelzens und Erstarrens wurden in Zeitkurven festgelegt und ergaben sich $z u \quad r 79,8^{\circ}$ (korr.) für die erstere und $180,2^{\circ}$ (korr.) für die letztere, also im Mittel I $80^{\circ}$, übereinstimmend mit Bunsens Beobachtung, während Kahlbaum I $86^{\circ}$ gefunden hat.

Da die Gewinnung der Bromwasserstoffsäure einfach und billig ist, auch der Preis des als Ausgangsmaterial dienenden Broms in neuester Zeit bedeutend zurückgegangen ist, da man ferner in dem nach der Elektrolyse zurückbleibenden Rest der Schmelze reines Litbiumsalz behält, während sonst ein nur schwcr zu trennendes Gemenge von Lithiumchlorid mit anderen Alkalimetallchloriden übrig bleibt und da das gewonnene Lithium vor allem ganz rein ist, so dürfte sich die Elektrolyse des Bromids unter Zusatz von 10 bis $15 \%$ Chlorid in der von uns angegebenen Weise zur Darstellung von Lithium in Laboratorien wobl empfehlen.

(Eingegangen: I2. Februar.)

\title{
ÜBER DEN PROPORTIONALITÄTSFAKTOR ZWISCHEN DEN BEWEGLICHKEITEN UND DEN ABSOLUTEN GESCHWINDIGKEITEN DER IONEN.
}

Von L. Bruner.

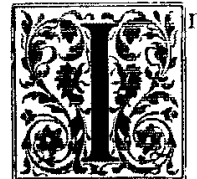

den meisten Lehrbüchern der physikalischen und der Elektrochemie pflegt man die Umrechnung der Ionenbeweglichkeiten $l_{A}$ und $l_{K}$ in absolute Ionengeschwindigkeit durch ziemlich schwerfällige und manchmal gekünstelte Denkoperationen zu gewinnen 1), obgleich z. B. bereits in Kohlrauschs "Leitvermögen der Elektrolyte" der entsprechende Gedankengang sich mit grosser Klarheit ausgesprochen befindet.

$\mathrm{Da}$ es sich in diesem Falle um die Bestimmung eines Proportionalitätsfaktors handelt, so dürfte es vielleicht nicht ohne pädagogischen Wert sein, denselben auf einem rein formellen Wege, nämlich durch Beachtung der Dimensionen der vorkommenden Grössen zu berechnen, wie dies so viclfach in anderen Gebieten der Physik üblich ist. Eine solche formolle Ableitung macht alle Denkkunststücke überflüssig, die trotz (oder vielleicht wegen)

I) Man vergleiche z. B. den sonst sehr lobenswerten „Leitfaden zum elektrotechnischen und elektrochemischen Seminar" von Berkitz und Roloff, S. I95. ihrer angestrebten Anschaulichkeit doch unbefriedigend erscheinen können.

Wir setzen

$$
\begin{gathered}
{\left[l_{K}\right]=[\mathrm{K} \varphi]=\frac{\mathrm{ccm} \cdot \mathrm{Aeq}}{\mathrm{Ohm} \cdot \mathrm{cm}}=\frac{\mathrm{Aeq} \cdot \mathrm{qcm}}{\mathrm{Ohm}}} \\
{[u]=\frac{\mathrm{cm} \cdot \mathrm{cm}}{\mathrm{sec} \cdot \text { Volt }}=\frac{\mathrm{qcm}}{\mathrm{sec} \cdot \text { Volt }}} \\
\psi=\frac{l_{K}}{u}=\frac{\mathrm{Aeq} \cdot \mathrm{qcm} \cdot \mathrm{sec} \cdot \text { Volt }}{\mathrm{Ohm} \cdot \mathrm{qcm}} \\
=\frac{\mathrm{Aeq} \cdot \mathrm{sec} \cdot \mathrm{Ohm} \cdot \mathrm{Amp} \cdot}{\text { Ohm }}=\mathrm{Aeq} \cdot \text { Coul. } \\
=96540 \text { Coul. }(F) .
\end{gathered}
$$

$\mathrm{Um}$ die Geschwindigkeiten der Ionen pro Volt $/ \mathrm{cm}$ Gefälle in $\mathrm{cm} / \mathrm{sec}$ zu erhalten, müssen wir also die Beweglichkeiten mit $9654^{\circ}$ Coul. dividieren.

Krakau. (Eingegangen: 17. Februar.)

1) Die Grössenbezeichnung schliesst sich der an, die in Kohlrauschs , Leitvermögen der Elektrolyte" benutzt ist. 